\title{
Viscoelastic material models for more accurate polyethylene wear estimation
}

\author{
Gioacchino Alotta', Olga Barrera ${ }^{2,3}$ and Elise C Pegg ${ }^{4}$
}

\begin{abstract}
Wear debris from ultra-high-molecular-weight polyethylene components used for joint replacement prostheses can cause significant clinical complications, and it is essential to be able to predict implant wear accurately in vitro to prevent unsafe implant designs continuing to clinical trials. The established method to predict wear is simulator testing, but the significant equipment costs, experimental time and equipment availability can be prohibitive. It is possible to predict implant wear using finite element methods, though those reported in the literature simplify the material behaviour of polyethylene and typically use linear or elastoplastic material models. Such models cannot represent the creep or viscoelastic material behaviour and may introduce significant error. However, the magnitude of this error and the importance of this simplification have never been determined. This study compares the volume of predicted wear from a standard elastoplastic model, to a fractional viscoelastic material model. Both models have been fitted to the experimental data. Standard tensile tests in accordance with ISO 527-3 and tensile creep recovery tests were performed to experimentally characterise both (a) the elastoplastic parameters and (b) creep and relaxation behaviour of the ultra-high molecular weight polyethylene. Digital image correlation technique was used in order to measure the strain field. The predicted wear with the two material models was compared for a finite element model of a mobile-bearing unicompartmental knee replacement, and wear predictions were made using Archard's law. The fractional viscoelastic material model predicted almost ten times as much wear compared to the elastoplastic material representation. This work quantifies, for the first time, the error introduced by use of a simplified material model in polyethylene wear predictions, and shows the importance of representing the viscoelastic behaviour of polyethylene for wear predictions.
\end{abstract}

\section{Keywords}

Polyethylene wear, material model, fractional viscoelasticity, unicompartmental knee arthroplasty, finite element analysis

Date received: 19 December 2017; accepted: 18 February 2018

\section{Introduction}

Wear of ultra-high-molecular-weight polyethylene (UHMWPE) components used for joint replacement prostheses can cause significant clinical complications, such as: implant loosening, osteolysis, inflammatory responses and post-operative pain. ${ }^{1}$ It is, therefore, essential to be able to predict implant wear as accurately as possible in vitro, to minimise the risk of unsafe implant designs continuing to clinical trials. The established method to predict the wear of an implant is with simulator testing. Wear simulator tests have been well characterised and validated against clinical data, and can predict implant wear to an acceptable degree of accuracy so is regularly used for validaton of new designs. ${ }^{2}$ However, wear simulator tests require significant equipment costs, availability of equipment is limited and the experiments take a long time. ${ }^{3}$
Numerical simulation provides an alternative method to predict wear. Maxian et al. ${ }^{4,5}$ were the first researchers to use discretisation to predict linear wear from a finite element (FE) model of an UHMWPE hip replacement component Maxian's work was based on a

\footnotetext{
'Bio/NanoMechanics for Medical Sciences Laboratory, ATeN-Center, Universita' degli Studi di Palermo, Palermo, Italy

${ }^{2}$ School of Engineering, Computing and Mathematics, Oxford Brookes University, Oxford, UK

${ }^{3}$ Nuffield Department of Orthopaedics, Rheumatology and

Musculoskeletal Sciences, University of Oxford, Oxford, UK

${ }^{4}$ Department of Mechanical Engineering, University of Bath, Bath, UK
}

\section{Corresponding author:}

Olga Barrera, School of Engineering, Computing and Mathematics, Oxford Brookes University, Wheatley Campus, Wheatley OX33 IHX, Oxon, UK.

Email: obarrera@brookes.ac.uk; olga.barrera@ndorms.ox.ac.uk 
study by Marshek and $\mathrm{Chen}^{6}$ who proposed that by applying Archard's wear equation to discrete elements of the articulating surfaces, non-uniform contact pressures and geometries could be taken into account. Maxian et al. applied Marshek's approach to FE models of an UHMWPE acetabular cup. The linear wear, or wear depth, $(\delta h)$ was calculated for each individual node on the articulating surface for each time increment $\left(\Delta t_{i}\right)$ from the contact stress $(\sigma)$, the sliding distance $(S)$ and the wear factor $\left(K_{w}\right)$ (equation (1)). Using this equation, the total wear for one cycle of loading was calculated for each node. To account for geometrical changes resulting from the wear, at a chosen number of cycles, the node positions are displaced by the calculated linear wear. The most reported studies apply a constant wear factor, but it has been shown that the wear factor of metal on UHMWPE varies depending on the contact stress. This limitation was addressed by Onişoru et al., ${ }^{7}$ who derived an equation to represent the relationship between contact stress and the wear factor, applied this to their wear calculations and reported an improved accuracy. Lui et al. ${ }^{8,9}$ used a similar approach but also took account of crossshearing effects to predict wear, based on the work by Kang et al. ${ }^{10}$

$$
\delta h_{\text {node }}=K_{w} \sum_{i=1}^{n} \sigma_{i} S_{i} \Delta t_{i}
$$

The majority of the reported numerical wear studies for UHMWPE use linear isotropic material models to represent the material behaviour (Table 1), which is a simplification of the behaviour of the material. The first study to calculate wear using a more complex material model for polyethylene was that of Teoh et al., ${ }^{12}$ who used a bilinear elastoplastic material representation. The authors reported an increase in contact stresses and wear with the elastoplastic model: the volumetric wear of the elastoplastic model was $57 \mathrm{~mm}^{3}$ year ${ }^{-1}$, over three times that of an elastic material model $\left(18 \mathrm{~mm}^{3}\right.$ year $\left.{ }^{-1}\right)$. Although an improvement, elastoplastic material models cannot represent the material behaviour, such as creep, stress relaxation, kinematic hardening or rate dependence, all of which are observed with polyethylene. Bevill et al. ${ }^{14}$ included creep behaviour in their wear calculations which enabled them to distinguish between linear wear and creep deformation giving valuable insight into the clinical scenario, and Lui et al. ${ }^{8,9}$ used a similar approach. However, neither study directly compared the difference a more representative material model for UHMWPE had on the predicted wear rate.

Viscoelastic material behaviour (creep, stress relaxation, as well as a 'fading' memory effect) can be represented by a combination of elastic behaviour (springs) and viscous behaviour (dashpots). The Maxwell or Kelvin-Voigt models are examples of spring and dashpot models; these have the advantage of fast implementation and can describe the time-dependent behaviour
Table I. Ultra-high-molecular-weight polyethylene material representation and wear calculations used in finite element wear analyses reported in the literature, where $K_{w}$ is the wear factor.

\begin{tabular}{|c|c|c|}
\hline Author & Material model & $K_{w}\left(\mathrm{~mm}^{3} \mathrm{~N}^{-1} \mathrm{~mm}^{-1}\right)$ \\
\hline Maxian et al. 4,5 & Linear elastic & $1.06 \times 10^{-9}$ \\
\hline Brown et al. ${ }^{\text {II }}$ & Linear elastic & $1.06 \times 10^{-9}$ \\
\hline Teoh et al. ${ }^{12}$ & Elastoplastic & $1.06 \times 10^{-9}$ \\
\hline Wu et al. ${ }^{13}$ & Linear elastic & $0.8 \times 10^{-9}$ \\
\hline Bevill et al. ${ }^{14}$ & Creep & $1.06 \times 10^{-9}$ \\
\hline Onişoru et al. ${ }^{7}$ & Linear elastic & $7.99 \sigma^{-0.653} \times 10^{-9}$ \\
\hline Fialho et al. ${ }^{15}$ & Linear elastic & $1.06 \times 10^{-9}$ \\
\hline Pal et al. ${ }^{16}$ & Elastoplastic & $2.64 \times 10^{-13}$ \\
\hline Kang et al. ${ }^{17}$ & Linear elastic & $1.24 \times 10^{-9}$ \\
\hline Lui et al. ${ }^{8}$ & Creep & $\mathrm{n} / \mathrm{a}$ \\
\hline Innocenti et al. ${ }^{18}$ & Linear elastic & $1.83 \times 10^{-14}$ \\
\hline Netter et al. ${ }^{3}$ & Linear elastic & $0.17 \times 10^{-9}$ \\
\hline
\end{tabular}

but cannot accurately represent polyethylene. Increasing complexity, with multiple springs and dashpots in different arrangements (such as Zener models), can capture the creep and relaxation behaviour but are computationally very demanding. An alternative approach is the use of fractional viscoelastic material models, which have been used successfully to represent very complex material properties for both short- and long-term time behaviour, such as polymers, rubbers, biological tissues and soils; ${ }^{19-22}$ these kinds of models have also been used to reproduce the behaviour of complex engineering components such as epoxy microbeam modelled with the FE method ${ }^{23-25}$ and the influence of temperature on the mechanical parameters has been investigated. ${ }^{26}$ Free energy and state expressions for power law relaxation/creep functions are discussed in Deseri and colleagues. ${ }^{27,28}$ A three-dimensional (3D) fractional viscoelastic theory has been derived and discussed in Alotta et al. ${ }^{29}$ Furthermore, the implementation in commercial FE software of a range of fractional viscoelastic models including fractional Maxwell, Kelvin-Voigt and Zener has been presented in Alotta et al. ${ }^{30}$ The purpose of this study was to investigate whether the application of a viscoelastic material model to represent UHMWPE in an FE model alters the predicted wear. A fractional viscoelastic material model was fit to experimentally derived data (which have not been presented elsewhere) and then applied to an FE model of a mobile unicompartmental knee replacement (The Oxford Knee, Zimmer-Biomet, Bridgend, UK) to examine the influence on wear. We report differences in the predicted wear for a simple ramp-loading scenario as a preliminary study, with a view to increasing the model complexity as future work.

\section{Materials and methods}

\section{Development of the fractional viscoelastic material model}

In classical viscoelasticity, the constitutive behaviour is obtained by combining the features of springs (elastic 
elements) and dashpots (viscous elements). The mechanical models obtained with this approach are characterised by exponential relaxation and creep functions. However, at the beginning of the twentieth century, it was observed that the creep and relaxation of many polymers is well fitted by power law functions ${ }^{19}$ (with power lying in the range $0-1$ ). In the frame of linear viscoelasticity, the Boltzmann superposition princi$\mathrm{ple}^{31}$ is assumed to be valid. If power law creep/ relaxation functions of the following types are assumed, the Boltzmann superposition principle leads directly to a constitutive law involving the so-called fractional operators (equations (2a) and (2b))

$$
\begin{aligned}
& R(t)=\frac{C_{\bar{\alpha}} t^{-\bar{\alpha}}}{\Gamma(1-\bar{\alpha})} \\
& C(t)=\frac{t^{\bar{\alpha}}}{C_{\bar{\alpha}} \Gamma(1+\bar{\alpha})}
\end{aligned}
$$

These are nothing but the integro-differential operators of real order defined as convolution integrals with power law kernel; ${ }^{32}$ in viscoelasticity, the order of integrals/derivatives is in the range $0-1$. Integral equations provide more general solutions with respect to differential equations; a more extended discussion on integral operators and integrability conditions may be found in Bongiorno. $^{33,34}$ In equation $(2), R(t)$ and $C(t)$ denote the creep and relaxation functions, respectively, $C_{\bar{\alpha}}$ and $\bar{\alpha}$ are the parameters, with $0 \leqslant \bar{\alpha} \leqslant 1$ and corresponding to the order of derivative (or integral), and $\Gamma(\cdot)$ is the Euler gamma function. The parameter $C_{\bar{\alpha}}$ is the viscoelastic modulus, the dimension of which is anomalous because it depends on the parameter $\bar{\alpha}$ that is a real number; the parameter $\bar{\alpha}$ controls the time scale and the shape of the creep and relaxation functions.

The most simple model is the springpot, often represented as a rhombus (see Figure 1). The constitutive equation of this model can be written as s5,36 $^{35}$

$$
\begin{aligned}
& \sigma(t)=C_{\bar{\alpha}}\left({ }^{C} D^{\bar{\alpha}} \varepsilon\right)(t) \\
& \varepsilon(t)=\frac{1}{C_{\bar{\alpha}}}\left(I^{\bar{\alpha}} \sigma\right)(t)
\end{aligned}
$$

where $\left({ }^{C} D^{\bar{\alpha}} \cdot\right)$ and $\left(I^{\bar{\alpha}} \sigma\right)$ are Caputo's fractional derivative and the Riemann-Liouville fractional integral, ${ }^{32}$ respectively. For simplicity of the notation, in the following Caputo's fractional derivative will be denoted simply by $\left(D^{\bar{\alpha}} \cdot\right)$.

The main advantage of the springpot model is that it is able to reproduce the power law behaviour observed experimentally and that it has long fading memory in agreement with the real behaviour of many materials. Moreover, it has been demonstrated that the behaviour of the springpot can be reproduced in classical viscoelasticity only by means of infinite sequence of springs and dashpots. ${ }^{37-39}$

It is to be noted that in equation (2) $R(0)=\infty$, $R(\infty)=0, C(0)=0$ and $C(\infty)=\infty$. However, experimental tests with many viscoelastic materials have

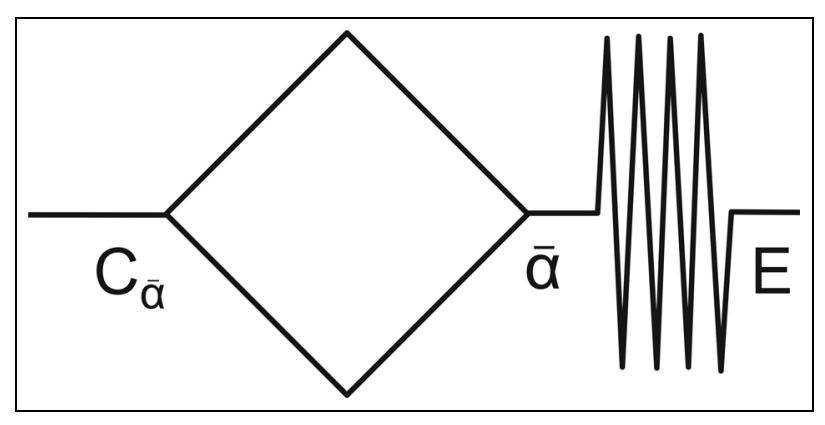

Figure I. Schematic illustration of the fractional Maxwell model.

revealed that often the relaxation and creep functions exhibit an initial $(t=0)$ and/or a long-term $(t \rightarrow \infty)$ finite value. For this reason, the springpot model is often used in combination with one or more springs. Experimental tests on UHMWPE considered in this work are well reproduced by a springpot in series with a spring, namely, a fractional Maxwell model (depicted in Figure 1). This result has been obtained by the authors in the experimental campaign described in the next section and is also confirmed by previous works. ${ }^{40}$ The constitutive law of the fractional Maxwell model is written as follows

$$
\left(D^{\bar{\alpha}} \sigma\right)(t)+\frac{E}{C_{\bar{\alpha}}} \sigma(t)=E\left(D^{\bar{\alpha}} \varepsilon\right)(t)
$$

where $E$ is Young's modulus related to the spring. The relaxation and creep functions of the fractional Maxwell model can be easily obtained as

$$
\begin{aligned}
& R(t)=E E_{\bar{\alpha}}\left(-\frac{E}{C_{\bar{\alpha}}} t^{\bar{\alpha}}\right) \\
& C(t)=\frac{1}{E}+\frac{t^{\bar{\alpha}}}{C_{\bar{\alpha}} \Gamma(1+\bar{\alpha})}
\end{aligned}
$$

with $E_{\bar{\alpha}}(\cdot)$ being the one-parameter Mittag-Leffler function defined as (see Podlubny ${ }^{32}$ )

$$
E_{\bar{\alpha}}(z)=\sum_{k=0}^{\infty} \frac{z^{k}}{\Gamma(1+\bar{\alpha} k)}
$$

Equations (4) and (5) are related to a unidimensional model; indeed, equation ( $5 \mathrm{~b}$ ) has been assumed as a basis for the fitting of the experimental test described in the next section. However, for the FE analysis a 3D model has to be defined. Assuming that the material is isotropic, only two relaxation or creep functions are needed in order to characterise the 3D behaviour of the material, one describing the pure volumetric behaviour and the other describing the pure shear behaviour. ${ }^{29,41,42}$ In compact form, the terms of the relaxation matrix are written as follows

$$
\begin{aligned}
R_{i j k h}(t)= & \left(K_{R}(t)-\frac{2}{3} G_{R}(t)\right) \delta_{i j} \delta_{k h} \\
& +G_{R}(t)\left(\delta_{i k} \delta_{j h}+\delta_{i h} \delta_{j k}\right)
\end{aligned}
$$




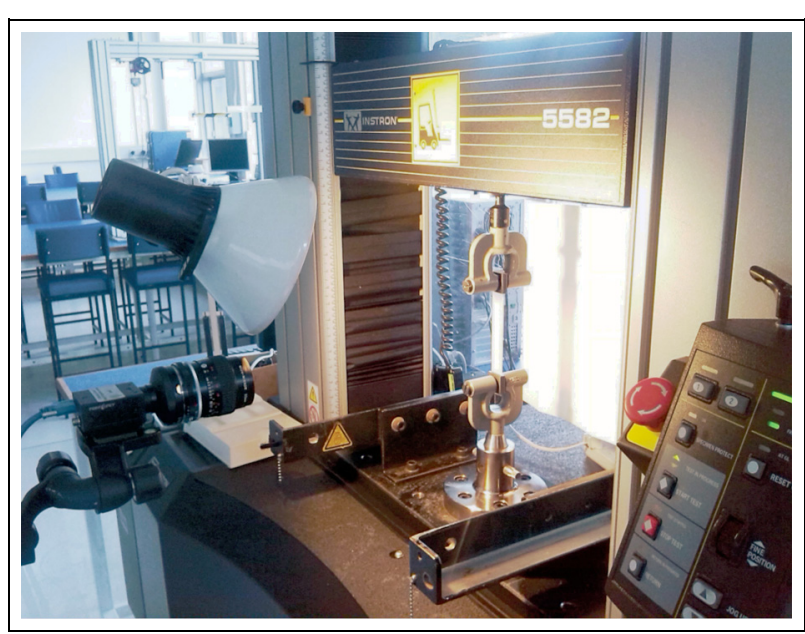

Figure 2. Experimental equipment used for the viscoelastic characterisation of the UHMWPE material.

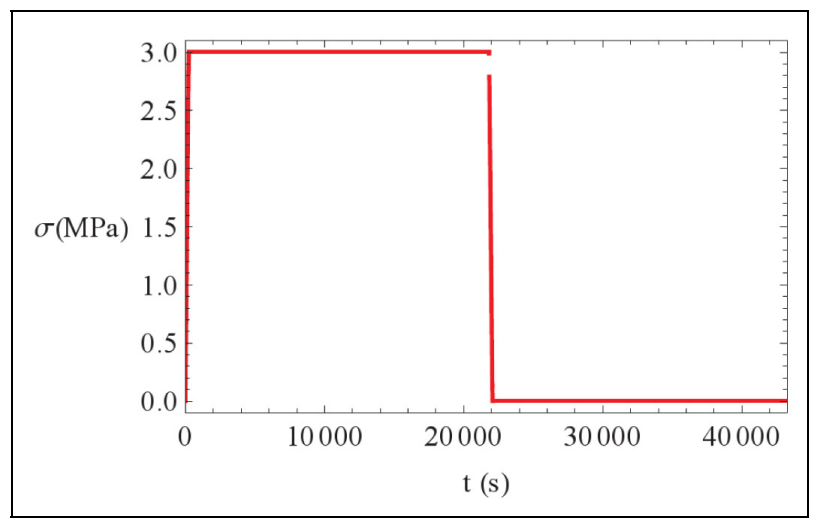

Figure 3. Variation of stress with time during the creep recovery test.

where $K_{R}(t)$ and $G_{R}(t)$ are the relaxation functions of the pure volumetric and pure shear components, respectively, and $\delta$ is the Kronecker delta. Assuming that both the components are well reproduced by fractional Maxwell models, the relaxation and creep functions are analogous to equation (5). The function related to the volumetric contribution is obtained from equation (5) by substituting $E, C_{\bar{\alpha}}$ and $\bar{\alpha}$ with $K, K_{\beta}$ and $\beta$, respectively. The function of the shear contributions are obtained from equation (5) by substituting $E, C_{\bar{\alpha}}$ and $\bar{\alpha}$ with $G, G_{\alpha}$ and $\alpha$, respectively.

In agreement with experimental evidence, the volumetric and shear time scales, which are determined by the parameters $\alpha$ and $\beta$, are not assumed equal. ${ }^{29,41,43}$ This allows the model to be very flexible and also to reproduce time-varying Poisson's ratio. ${ }^{29}$

\section{Experimental determination of UHMWPE viscoelastic parameters}

Uniaxial creep recovery experimental tests were performed to characterise the parameters to use for the viscoelastic material model. Tensile test specimens were machined from in-house sheet moulded UHMWPE, made from GUR 4150 resin (Celanese, Frankfurt am Main, Germany), which is the non-medical grade equivalent of GUR 1050. The samples were machined to a rectangular geometry of $180 \mathrm{~mm} \times 20 \mathrm{~mm} \times 1 \mathrm{~mm}$. The strain in the direction of the applied stress was measured using digital image correlation (DIC).

Tensile tests were performed on an electromechanical test machine (5582; Instron, Norwood, MA, US) (Figure 2). The choice to perform mechanical tests in tension is dictated by the fact that in tension it is possible to use long specimens that experience displacements larger than compact specimen for compression, so that DIC measurements are more reliable in tension. On the other hand, although the behaviour in compression may be slightly different than in tension, we assumed a linear viscoelastic behaviour of the material, then under this hypothesis the behaviour in tension and compression are equal.

Different magnitudes of the constant applied stress $\left(\sigma_{0}\right)$ load were applied: 1,3 and $5 \mathrm{MPa}$; five specimens were tested for each stress level. The parameters obtained by the fitting of experimental data at different levels of stress were homogeneous. For this reason, the material can be considered linearly viscoelastic at the least up to $5 \mathrm{MPa}$ of applied stress. This fact was confirmed by the results published in Mourad et al. ${ }^{44}$ where it is shown that UHMWPE may be considered linear up to $10 \mathrm{MPa}$.

The maximum tensile stress $\sigma_{0}$ was reached after $4 \mathrm{~min}$ of ramp loading. The stress was maintained for $6 \mathrm{~h}$, after which the load was reduced to zero over a period of $4 \mathrm{~min}$, and the samples were left to recover for $6 \mathrm{~h}$ (Figure 3).

The fitting of experimental data has taken into account the exact history of stress described above and to this purpose it is written as

$$
\begin{aligned}
\sigma(t) & =\frac{\sigma_{0}}{t_{0}}\left\{\left[t-\left(t-t_{0}\right) U\left(t-t_{0}\right)\right]\right. \\
& \left.-\left[\left(t-t_{1}\right) U\left(t-t_{1}\right)-\left(t-t_{2}\right) U\left(t-t_{2}\right)\right]\right\}
\end{aligned}
$$

where $t_{0}=4 \mathrm{~min}$ is the time at the end of the loading ramp, $t_{1}=364 \mathrm{~min}$ is the time at the end of the creep phase, $t_{2}=368 \mathrm{~min}$ is the time at the end of the unloading ramp and $U(t)$ is the unit-step function. By assuming the creep function of equation (5b), the history of stress of equation (8) generates the following theoretical history of strain (Figure 4) that was used to fit experimental test

$$
\begin{aligned}
\epsilon(t)= & \frac{\sigma_{0}}{E t_{0}}\left\{\left[t-\left(t-t_{0}\right) U\left(t-t_{0}\right)\right]\right. \\
& \left.-\left[\left(t-t_{1}\right) U\left(t-t_{1}\right)-\left(t-t_{2}\right) U\left(t-t_{2}\right)\right]\right\} \\
& +\frac{\sigma_{0}}{C_{\bar{\alpha}} t_{0}}\left\{\left[t^{1+\bar{\alpha}}+-\left(t-t_{0}\right)^{1+\bar{\alpha}}+U\left(t-t_{0}\right)\right]\right. \\
& \left.-\left[\left(t-t_{1}\right)^{1+\bar{\alpha}} U\left(t-t_{1}\right)-\left(t-t_{2}\right)^{1+\bar{\alpha}}+U\left(t-t_{2}\right)\right]\right\}
\end{aligned}
$$

The values of the obtained parameters $E, C_{\bar{\alpha}}$ and $\bar{\alpha}$ are reported in Table 3. The parameters related to the 


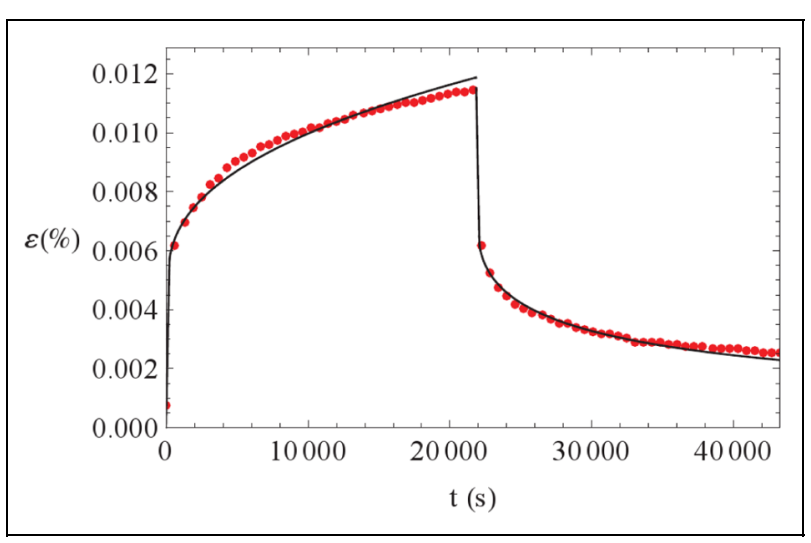

Figure 4. Variation of strain with time during the creep recovery test. Test results are indicated dotted line and the theoretical curve with the fitted parameters is shown with continuous line.

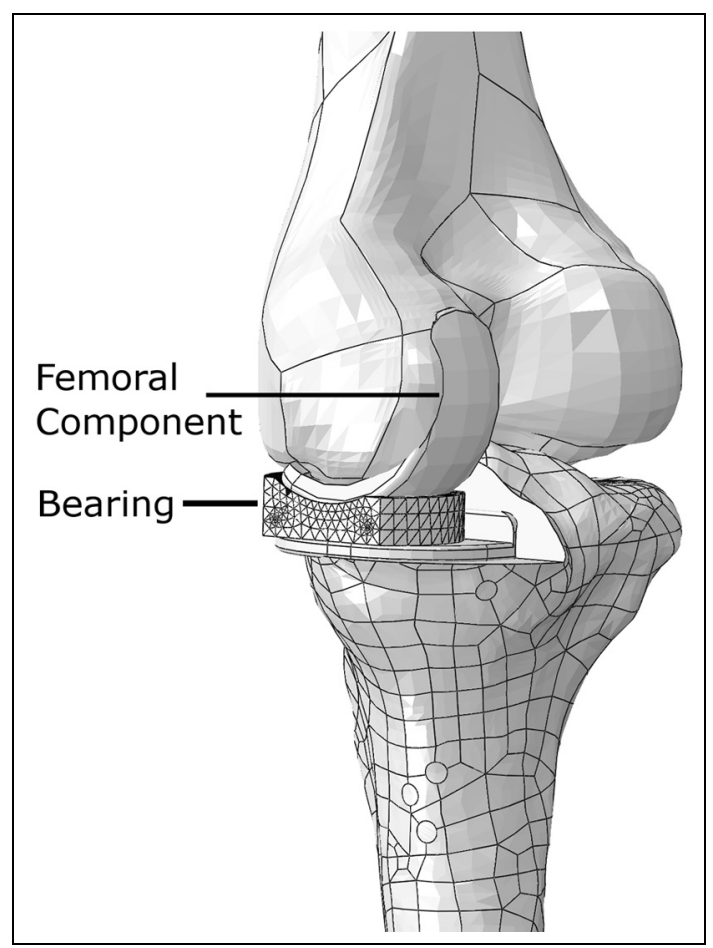

Figure 5. Illustration of the finite element model assembly, where the meshed bearing and articulating femoral components are shown in the context of the knee. The femur, tibia and tibial component did not contribute to the model, but are included for illustrative purposes.

Table 2. Plastic material properties defined for the elastoplastic models.

\begin{tabular}{ll}
\hline True stress $(\mathrm{MPa})$ & True plastic strain \\
\hline 2.8 & 0.00 \\
9.2 & 0.01 \\
13.5 & 0.02 \\
16.4 & 0.03 \\
18.3 & 0.04 \\
21.7 & 0.07 \\
\hline
\end{tabular}

3D constitutive law $\left(G, G_{\alpha}, \alpha, K, K_{\beta}\right.$ and $\left.\beta\right)$ are reported in Table 4 . These have been obtained by considering a constant Poisson's ratio $\nu=0.46$ (the value commonly considered for UHMWPE) and the following well-known relationships have been used

$$
\begin{aligned}
& G=\frac{E}{2(1+\nu)} \\
& K=\frac{E}{3(1-2 \nu)}
\end{aligned}
$$

Analogous relationships have been used to obtain $G_{\alpha}$ and $K_{\beta}$ from $C_{\bar{\alpha}}$. The hypothesis of constant Poisson's ratio implies also that $\alpha=\beta=\bar{\alpha}$; this means that the volumetric and shear contributions evolve with the same time scale in our FE model. This fact is in disagreement with the experimental results obtained in the past studies with other polymers ${ }^{41,43}$ and it is possible that also for UHMWPE the time scales of the two contributions are different. However, the direct determination of the two time scales may be performed only if in the uniaxial creep test we are able to measure correctly not only the longitudinal strain but also the transverse strain. Another strategy is to perform two different creep tests, for example, a uniaxial creep test and a torsion creep test. In this work, it has not been possible to perform a double measure in the uniaxial creep test. However, for the scope of the work, that is, to compare the predicted wear with a commonly used elastoplastic model and with a fractional viscoelastic model, this approximation is acceptable.

\section{FE model definition}

The FE model consisted of a UHMWPE unicompartmental knee bearing component (The Oxford Partial Knee, Zimmer-Biomet) and an articulating femoral component modelled as an analytical rigid body. A medium-sized component was modelled, as this is the size most commonly implanted; drawings of the component geometries have been previously published. ${ }^{45}$ The femoral component was a sphere of radius $24 \mathrm{~mm}$, cut to a width of $20 \mathrm{~mm}$. The upper articulating surface of the bearing conformed to the femoral component with a clearance of $0.2 \mathrm{~mm}$. The thickness of the bearing in the centre was $3.5 \mathrm{~mm}$, and the bearing was $34 \mathrm{~mm}$ long by $24 \mathrm{~mm}$ wide. Holes for marker wires were included and positioned $3 \mathrm{~mm}$ from the base of the bearing, and the marker wires themselves were represented as rigid cylinders of $1 \mathrm{~mm}$ diameter.

The components were assembled as shown in Figure 5; the femur, tibia and tibial component did not contribute to the model but are included for illustrative purposes. The load was applied axially to the femoral component, perpendicular to the base of the bearing. The component was compressively ramp loaded to $1200 \mathrm{~N}$ over a period of $0.2 \mathrm{~s}$, representing average loading during a step-up activity. ${ }^{46}$ The base of the bearing was constrained in the axial direction. Contact was defined between the femoral 


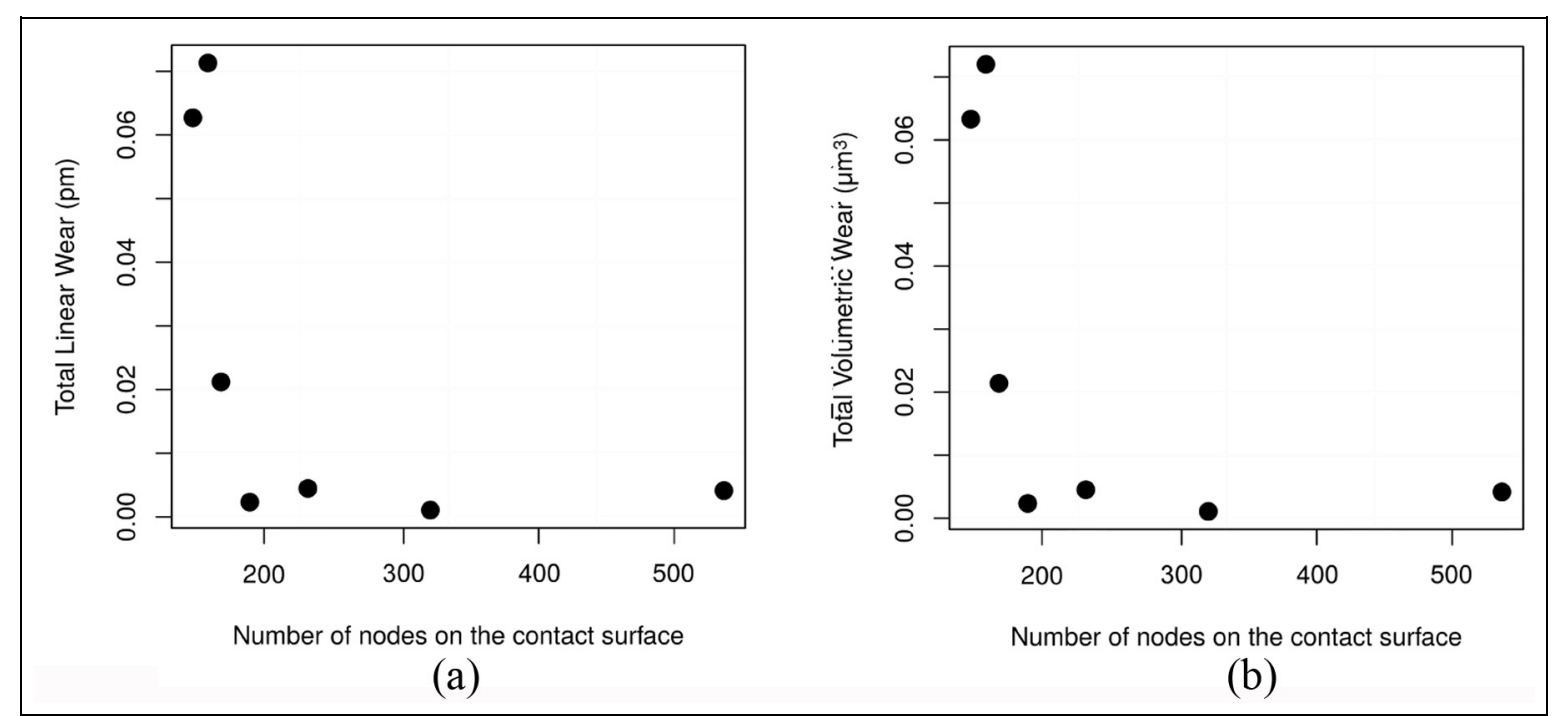

Figure 6. Variation of the calculated wear for different mesh densities. A mesh size of $3.5 \mathrm{~mm}$ was deemed converged (I89 nodes): (a) linear wear and (b) volumetric wear.

component and the upper surface of the bearing, and a stiffness (penalty) contact algorithm was used with finite sliding and a friction coefficient of $0.08 .^{47}$ Tie constraints were used to fix the marker wires within the bearing. The bearing was meshed with quadratic tetrahedral elements (C3D10M), and the converged mesh size was used, the determination of which is described in the section on mesh convergence.

The only material property assigned to the metallic components was density, as these were modelled as rigid bodies. The femoral component was modelled with a density of $8.387 \mathrm{~g} \mathrm{~cm}^{-3}$ to represent the $\mathrm{Co}-\mathrm{Cr}-$ Mo alloy, ${ }^{48}$ and the marker wires were assigned a density of $4.42 \mathrm{~g} \mathrm{~cm}^{-3}$ for Ti6-A14-V alloy. ${ }^{49}$ A subroutine was created to apply the fractional viscoelastic model described in the previous section. The input parameters used for the elastoplastic material model were determined from standard tensile test results, where the sheet moulded GUR 4150 material was tested in accordance with ISO 527-3 using Specimen Type 2 geometry. The calculated parameters were a modulus of $855.2 \mathrm{MPa}$ and a Poisson's ratio of 0.46 , and the plasticity parameters are summarised in Table 2. The material behaviour of the UHMWPE was assumed to be the same in compression as in tension for both models.

All models were created, solved and post-processed using the FE software Abaqus (version 6.12, Dassault Systémes, Paris, France). An explicit solver was used with an imposed time increment of $4 \times 10^{-6}$, and validation of the mass scaling has been described previously. ${ }^{45}$

\section{Quantification of wear}

Linear wear was calculated for the two different models using equation (1). A wear factor of $1.06 \times 10^{-9} \mathrm{~mm}^{3} \mathrm{~N}^{-1} \mathrm{~mm}^{-1}$ was used, as reported by Maxian et al. ${ }^{4,5}$ The linear wear for each time increment was the maximum linear wear of all the nodes on the articulating surface. The volumetric wear was the sum of the linear wear of all the nodes on the articulating surface multiplied by the surface area $\left(766.2 \mathrm{~mm}^{2}\right)$.

The sliding distance $(S)$ was calculated using the great-circle distance equation (11), which assumed that the sliding occurred around the circumference of the femoral component. The cartesian coordinates of the position of the nodes at the start and the end of the increment were converted to polar coordinates $\left(\phi_{1}, \lambda_{1}\right.$ and $\phi_{2}, \lambda_{2}$, respectively) relative to the centre of the femoral component. The femoral component radius $(24 \mathrm{~mm})$ was used as the sphere radius $(R)$, as the articulating surface of the design is spherical

$$
\begin{aligned}
& S=2 R \sin ^{-1} \\
& {\left[\sin ^{2}\left(\frac{\phi_{2}-\phi_{1}}{2}\right)+\cos \left(\phi_{1}\right) \cos \left(\phi_{2}\right) \sin ^{2}\left(\frac{\lambda_{2}-\lambda_{1}}{2}\right)\right]^{0.5}}
\end{aligned}
$$

\section{Mesh convergence}

The mesh convergence was performed for the linear wear and volumetric wear output. The mesh seeding densities examined ranged from 2.0 to $5.0 \mathrm{~mm}$, with 0.5-mm intervals, which created between 115 and 526 nodes on the articular surface. Both the linear wear and volumetric wear converged at a mesh size of $3.5 \mathrm{~mm}$ (Figure 6). Convergence was defined as when the result was within $30 \%$ of the next three smaller mesh sizes.

\section{Results}

\section{Definition of the fractional viscoelastic material model}

The results of the creep recovery experimental tests were fitted to the fractional viscoelastic Maxwell model 
Table 3. Parameters determined from the creep recovery test results to represent GUR 4I50 UHMWPE.

\begin{tabular}{ll}
\hline Parameter & Value \\
\hline $\bar{\alpha}$ & 0.4 \\
$C_{\bar{\alpha}}$ & $24553 \mathrm{MPas} \cdot \bar{\alpha}$ \\
$E$ & $561 \mathrm{MPa}$ \\
\hline
\end{tabular}

Table 4. Input parameters for the fractional Maxwell model, identified from the creep recovery test results.

\begin{tabular}{ll}
\hline Parameter & Value \\
\hline$K$ & $2338 \mathrm{MPa}$ \\
$G$ & $192 \mathrm{MPa}$ \\
$K_{\beta}$ & $102304 \mathrm{MPa} \cdot s^{\beta}$ \\
$G_{\alpha}$ & $8404 \mathrm{MPa} \cdot \mathrm{s}^{\alpha}$ \\
$\alpha$ & 0.4 \\
$\beta$ & 0.4 \\
\hline
\end{tabular}

as shown in Figure 4. The fitted parameters are summarised in Table 3. It can be seen that the parameters were of a good fit to the experimental data. These data were then converted into the parameters necessary for the fractional viscoelastic model as described in the previous section, and these are summarised in Table 4.

\section{Wear volume prediction}

The wear prediction (for both linear and volumetric wear) using the fractional viscoelastic material model to represent UHMWPE was almost 10 times greater than that predicted using an elastoplastic material model (Figure 7). When the wear factor was calculated using the Onişoru equation (7), this difference was even greater but the overall predicted wear was reduced.
The cumulative increase in wear (both linear and volumetric) was approximately linear for the elastoplastic material model (coefficient of determination $=$ 0.912 for linear wear and 0.834 for volumetric wear). However, the viscoelastic model deviated from linearity at higher loads (Figures 8 and 9).

\section{Stress analysis}

The overall stress within the bearing was increased when the UHMWPE was represented as a viscoelastic material, but in particular a difference was noticed in the stress on the contact surface and in the contact region. Figure 10 illustrates a cross-section through the centre of the bearing for the two different material models. It can be seen that in the viscoelastic model the stress is more concentrated around the articulating surface, whereas in the elastoplastic material model the stress is evenly distributed through the thickness of the bearing.

\section{Discussion}

The results of this study have demonstrated a clear difference in the wear prediction from an FE model of a UHMWPE component when using a viscoelastic material model definition compared with an elastoplastic model. It is known that elastoplastic material models will underestimate stress due to the stress-relieving effect of plasticity. However, numerous authors have used linear elastic material models to predict wear and the results have correlated well with with either experimental wear test data or clinical data. It is therefore unexpected that a more representative material model can have such a large influence on the predicted wear.

One possible reason for this discrepancy could be the wear factor $K_{w}$. As shown in Table 1, a wide range

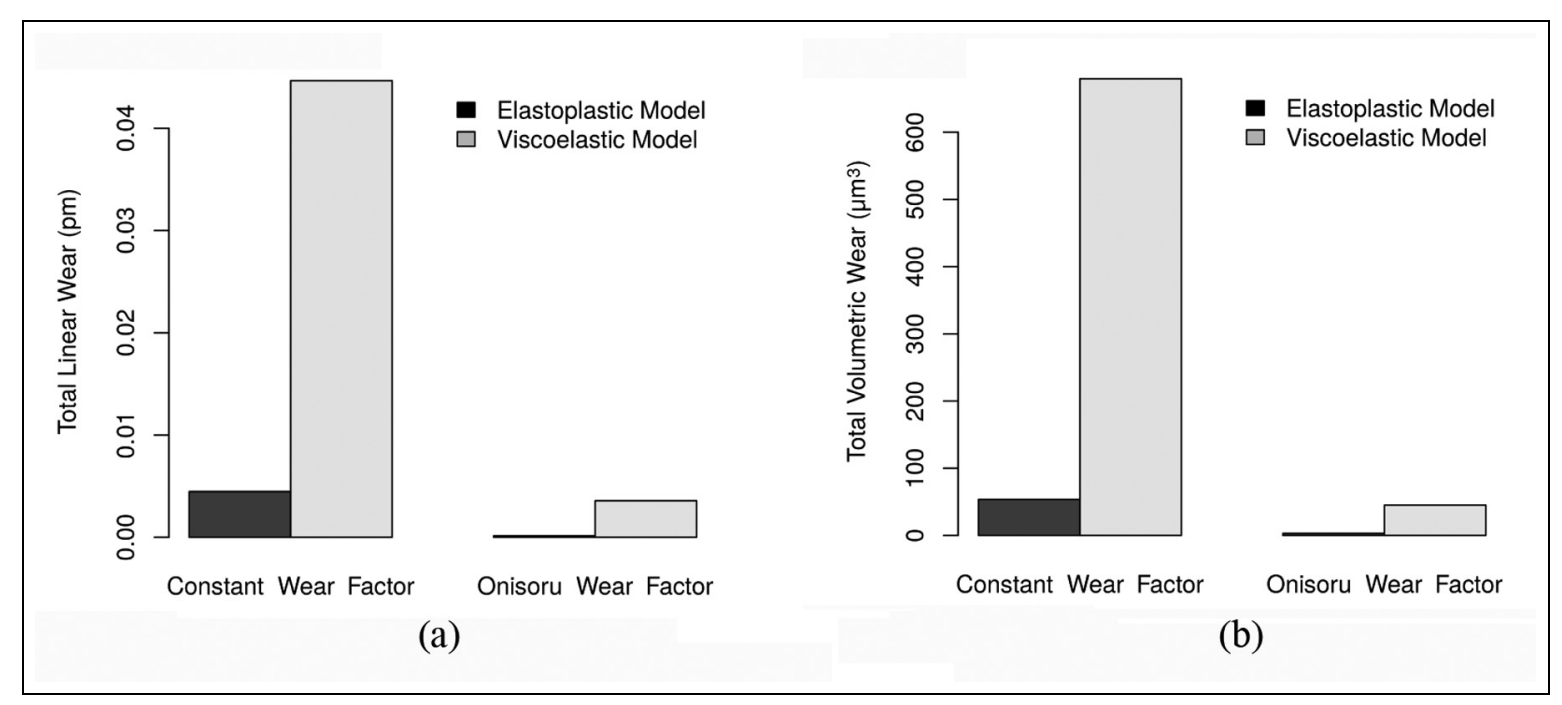

Figure 7. Calculated total linear (a) and volumetric (b) wear for the elastoplastic material model and the fractional elastic material model. Results were obtained using a constant wear factor of $1.06 \times 10^{-9} \mathrm{~mm}^{3} \mathrm{~N}^{-1} \mathrm{~mm}^{-1}$ and the Onisoru wear factor which used a variable wear factor calculated from the contact stress $\left(7.99 \sigma^{-0.653}\right)$. 


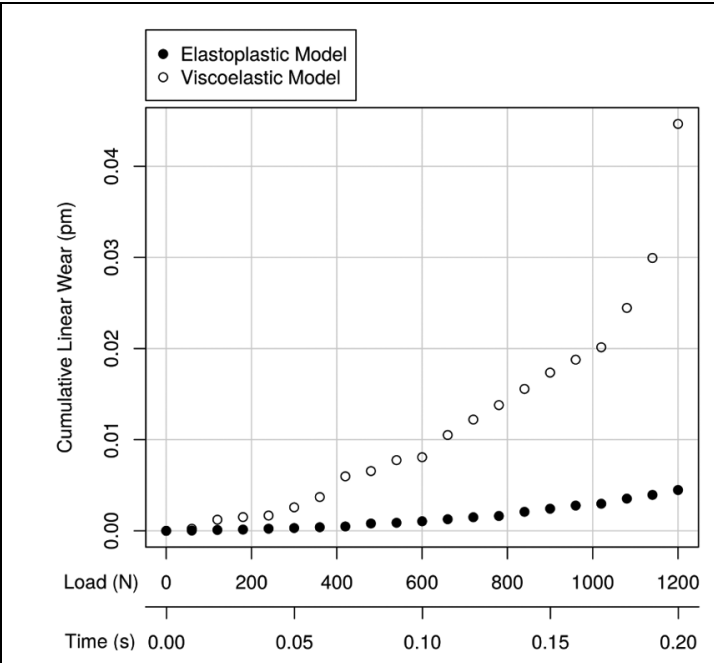

(a)

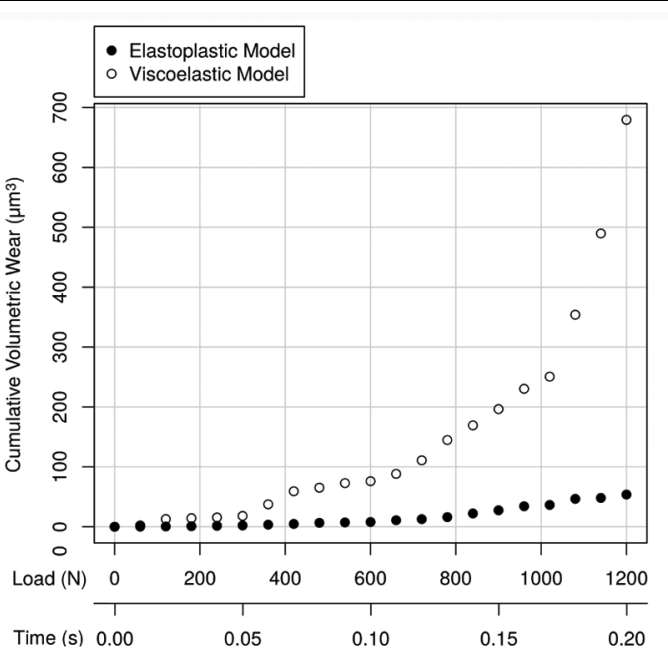

(b)

Figure 8. Cumulative wear calculated throughout the loading step. Results are shown for the elastoplastic material model and the fractional viscoelastic material model, calculated using a constant wear factor of $1.06 \times 10^{-9} \mathrm{~mm}^{3} \mathrm{~N}^{-1} \mathrm{~mm}^{-1}$ : (a) linear wear and (b) volumetric wear.

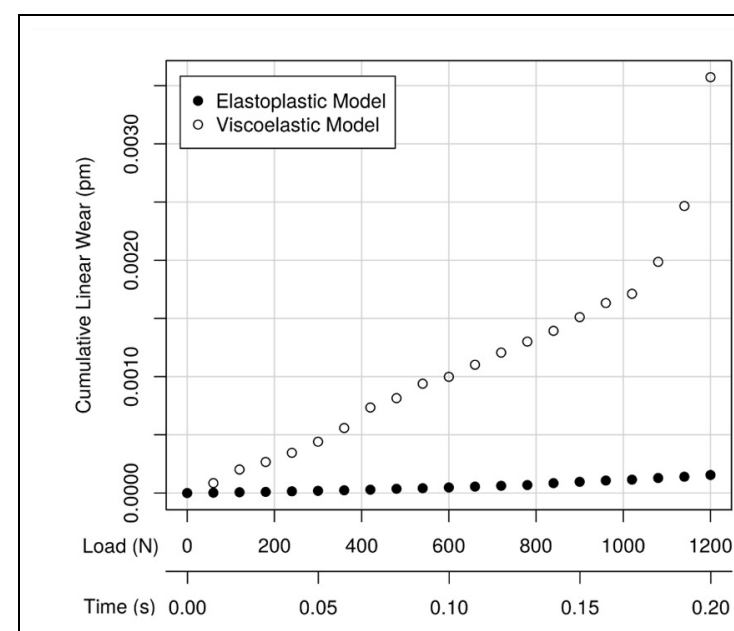

(a)

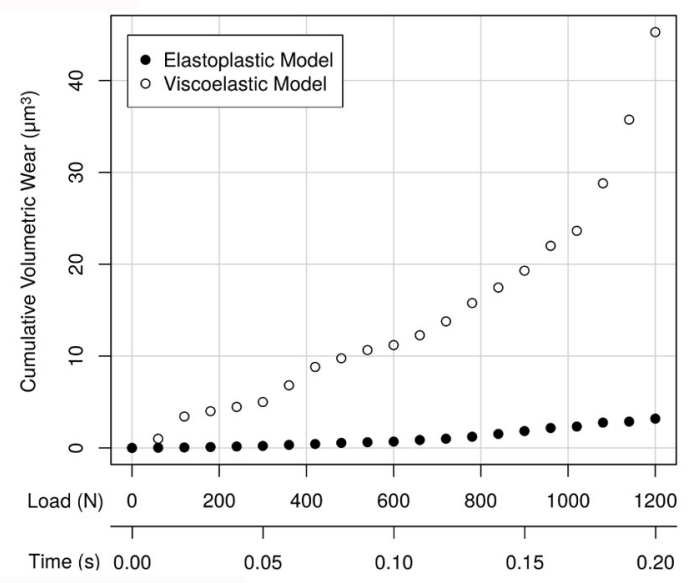

(b)

Figure 9. Cumulative wear calculated throughout the loading step. Results are shown for the elastoplastic material model and the fractional viscoelastic material model, calculated using a variable wear factor calculated from the contact stress $\left(9.77 \sigma^{-0.653}\right)$ :

(a) linear wear and (b) volumetric wear.

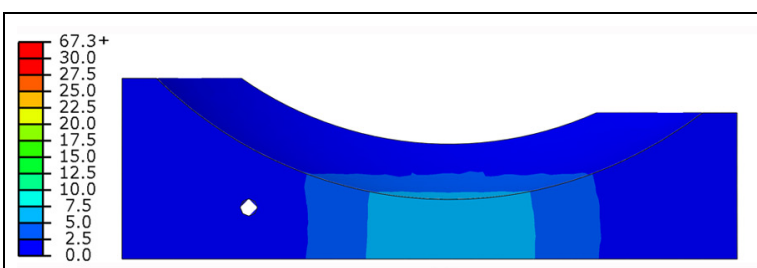

(a)

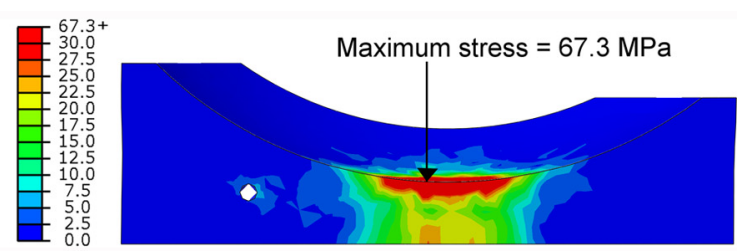

(b)

Figure 10. Cross-sectional view through the centre of the unicompartmental knee bearing in the sagittal plane. The von Mises stress ( $\mathrm{MPa}$ ) distribution within the bearing is illustrated for the results using the different material models, where the colour key ranges from 0 to $30 \mathrm{MPa}$ and the position of maximum stress is highlighted: (a) elastoplastic material model and (b) fractional viscoelastic model. 
of wear coefficients are reported in the literature; the values range from $0.00002 \times 10^{-9}$ to $1.2 \times 10^{-9} \mathrm{~mm}^{3} \mathrm{~N}^{-1} \mathrm{~mm}^{-1}$. The majority of wear factors are calculated from pin-on-disc experiments which can have a simplified loading scenario compared to in vivo loading, but some studies have used simulator wear results and derived data clinically to calculate wear factors ${ }^{3}$ which are likely to be more representative. Nevertheless, there is a need for more research to accurately determine the wear factor of metal on UHMWPE for different situations to ensure the accuracy of numerical wear predictions.

Despite being a more accurate representation of the material behaviour, it may be that the increased wear predicted by the viscoelastic model is not representative of reality. UHMWPE is known to harden due to alignment of molecular chains under cyclic loading and will also oxidise over time in vivo. Neither the viscoelastic model nor the elastoplastic model takes into account the hardening. Including hardening effects into the model would reduce the wear rate. It could be that the inclusion of kinematic hardening into the material model or alteration of the wear factor with loading cycles could create a more realistic prediction of wear. Use of the wear factor to represent the so-called 'running in wear' was reported by Liu et al., ${ }^{50}$ who examined wear of metal-on-metal hip replacements using FE analysis. The wear was calculated by defining two wear coefficients, one for short-term wear and the other for long-term wear. It may be possible to use a similar methodology to represent hardening and subsurface oxidation of UHMWPE with time while maintaining computational efficiency.

Another factor to consider in the wear calculation is the determination of the sliding distance. In this study, the sliding distance was calculated using the great-circle distance equation, which was possible due to the conforming nature of the articulating surfaces and the spherical geometry. In the design of the Oxford Unicompartmental Knee, there is a $0.2-\mathrm{mm}$ clearance between the femoral component and the bearing. In this study, because the femoral component was modelled as a rigid part, it was valid to assume that, where contact occurred on the bearing surface, this clearance must have been closed by deformation of the bearing. However, if the material properties had been assigned to the femoral component, the use of the great-circle distance equation could have introduced errors. Studies in the literature often do not mention how sliding distance has been calculated. Teoh et al. mention using the great-circle distance equation to calculate the sliding distance. Other studies calculate the sliding distance based upon a defined rotational or translational displacement, ${ }^{13}$ but these assume no change in the component geometry. However, the influence of this assumption would be expected to be minor in the case of large displacements and small wear.

In this study, the fractional viscoelastic model predicted approximately 10 times more linear wear compared to the elastoplastic material model, and over one loading cycle the difference in magnitude of predicted wear was $0.04 \mathrm{pm}$. A patient after knee arthroplasty typically walks 1 million steps, and so in 1 year the difference in predicted linear wear would be $0.04 \mu \mathrm{m}$ (assuming a linear increase in wear with time). This represents a large difference clinically in terms of both UHMWPE wear particles within the joint and damage to the component.

\section{Conclusion}

In conclusion, this study has shown that the use of simplified material models to represent polyethylene to predict wear introduces significant (up to 10 times) error in the calculated wear volume. In contrast, the fractional viscoelastic material model, which was defined from the experimental data, predicted concentrated stresses on the articulating surface, which matches well with the damage observed in the retrieved components. ${ }^{51}$ Use of such accurate material models in FE models of joint replacements could prove to be a cost-efficient and reliable way to predict wear and aid optimal implant design.

\section{Acknowledgements}

G.A. wishes to acknowledge support from the University of Palermo to visit the University of Oxford, during which period this research was conducted. O.B. and E.C.P. designed the study. G.A. and O.B. performed the mechanical testing and created the fractional viscoelastic material model. E.C.P. developed the finite element model and G.A. implemented the fractional viscoelastic material code. E.C.P. performed the data analysis and wrote the paper and O.B. and G.A. edited the manuscript.

\section{Declaration of conflicting interests}

The author(s) declared no potential conflicts of interest with respect to the research, authorship and/or publication of this article.

\section{Funding}

The author(s) received no financial support for the research, authorship and/or publication of this article.

\section{References}

1. Goodman SB. Wear particles, periprosthetic osteolysis and the immune system. Biomaterials 2007; 28: 5044 5048.

2. Essner A, Schmidig G and Wang A. The clinical relevance of hip joint simulator testing: in vitro and in vivo comparisons. Wear 2005; 259: 882-886.

3. Netter J, Hermida J, Flores-Hernandez C, et al. Prediction of wear in crosslinked polyethylene unicompartmental knee arthroplasty. Lubricants 2015; 3: 381-393. 
4. Maxian TA, Brown TD, Pedersen DR, et al. A slidingdistance-coupled finite element formulation for polyethylene wear in total hip arthroplasty. $J$ Biomech 1996; 29: 687-692.

5. Maxian TA, Brown TD, Pedersen DR, et al. Adaptive finite element modeling of long-term polyethylene wear in total hip arthroplasty. J Orthop Res 1996; 14: 668-675.

6. Marshek FM and Chen HH. Discretization pressurewear theory for bodies in sliding contact. J Tribol 1989; 111: 95-101.

7. Onişoru J, Capitanu L and Iarovici A. Prediction of wear of acetabulum inserts due to multiple human routine activities. Tribology 2006; 8: 28-33.

8. Lui F, Fisher $\mathbf{J}$ and Jin Z. Computational modelling of polyethylene wear and creep in total hip joint replacements: effect of the bearing clearance and diameter. Proc IMechE, Part J: J Engineering Tribology 2012; 226: 551563.

9. Lui F, Fisher $\mathbf{J}$ and Jin Z. Effect of motion inputs on the wear prediction of artificial hip joints. Tribology Int 2013; 63: 105-114.

10. Kang L, Galvin AL, Brown TD, et al. Quantification of the effect of cross-shear on the wear of conventional and highly cross-linked UHMWPE. J Biomech 2008; 41: 340 346.

11. Brown TD, Steward KJ, Nieman JC, et al. Local head roughening as a factor contributing to variability of total hip wear: a finite element analysis. J Biomech Eng 2002; 124: 691-698.

12. Teoh SH, Chan WH and Thampuran R. An elasto-plastic finite element model for polyethylene wear in total hip arthroplasty. J Biomech 2002; 35: 323-330.

13. Wu JS-S, Hung J-P, Shu C-S, et al. The computer simulation of wear behaviour appearing in total hip prosthesis. Comput Meth Prog Bio 2003; 70: 81-91.

14. Bevill SL, Bevill GR, Penmetsa JR, et al. Finite element simulation of early creep and wear in total hip arthroplasty. J Biomech 2005; 38: 2365-2374.

15. Fialho JC, Fermandes PR, Eça L, et al. Computational hip joint simulator for wear and head generation. $J$ Biomech 2007; 40: 2358-2366.

16. Pal S, Haider H, Laz PJ, et al. Probabilistic computational modeling of total knee replacement wear. Wear 2008; 264: 701-707.

17. Kang L, Galvin AL, Fisher J, et al. Enhanced computational prediction of polyethylene wear in hip joints by incorporating cross-shear and contact pressure in additional to load and sliding distance: effect of head diameter. J Biomech 2009; 42: 912-918.

18. Innocenti B, Labey L, Kamali A, et al. Development and validation of a wear model to predict polyethylene wear in total knee arthroplasty: a finite element analysis. Lubricants 2014; 2: 193-205.

19. Nutting PG. A new general law of deformation. J Frankl Inst 1921; 191: 679-685.

20. Bagley RL and Torvik PJ. On the appearance of the fractional derivative in the behaviour of real materials. $J$ Appl Mech 1984; 51: 294-298.

21. Deseri L, Di Paola M, Zingales M, et al. Power-law hereditariness of hierarchical fractal bones. Int $J$ Numer Meth Bio 2013; 29(12): 1338-1360.

22. Alotta G, Di Paola M and Pirrotta A. Fractional TajimiKanai model for simulating earthquake ground motion. B Earthq Eng 2014; 12: 2495-2506.
23. Alotta G, Failla G and Zingales M. Finite-element formulation of a non-local hereditary fractional-order Timoshenko beam. ASCE: J Eng Mech 2017; 143(5): D4015001.

24. Alotta G, Failla G and Pinnola FP. Stochastic analysis of a non-local fractional viscoelastic bar forced by Gaussian white noise. ASCE-ASME J Risk Uncertainty, Part B: Mech Eng 2017; 3(3): 030904.

25. Alotta G, Di Paola M, Failla G, et al. On the dynamics of non-local fractional viscoelastic beams under stochastic agencies. Compos Part B: Eng 2018; 137: 102-110.

26. Alotta $G$ and Colinas-Armijo N. Analysis of fractional viscoelastic material with mechanical parameters dependent on random temperature. ASCE-ASME J Risk Uncertainty, Part B: Mech Eng 2017; 3(3): 030906.

27. Deseri L, Di Paola M and Zingales M. Free energy and states of fractional-order hereditariness. Int $J$ Solids Struct 2014; 51(18): 3156-3167.

28. Deseri L, Zingales $M$ and Pollaci P. The state of fractional hereditary materials. Discrete Cont Dyn-B 2014; 19(7): 2065-2089.

29. Alotta G, Barrera O, Cocks ACF, et al. On the behaviour of a three-dimensional fractional viscoelastic constitutive model. Meccanica 2016; 52(9): 2127-2142.

30. Alotta G, Barrera O, Cocks ACF, et al. The finite element implementation of 3D fractional viscoelastic constitutive models. In: Finite element analysis and design, 2018.

31. Christensen RM. Theory of viscoelasticity: an introduction. New York: Academic Press, 1982.

32. Podlubny I. Fractional differential equation. San Diego, CA: Academic Press, 1999.

33. Bongiorno D. Metric differentiability of Lipschitz maps. J Aust Math Soc 2014; 96: 25-35.

34. Bongiorno D. On the problem of regularity in the Sobolev space. Topol Appl 2009; 156(18): 2986-2995.

35. Gemant A. A method of analyzing experimental results obtained from elasto-viscous bodies. J Appl Phys 1936; 7: 311-317.

36. Scott-Blair GW and Caffyn JE. An application of the theory of quasi-properties to the treatment of anomalous strain-stress relations. Philos Mag 1949; 40: 80-94.

37. Schiessel $\mathrm{H}$ and Blumen A. Hierarchical analogues to fractional relaxation equations. $J$ Phys A: Math Gen 1993; 26: 5057-5069.

38. Di Paola M and Zingales M. Exact mechanical models of fractional hereditary materials. $J$ Rheol 2012; 56(5): 9831004.

39. Di Paola M, Pinnola FP and Zingales M. A discrete mechanical model of fractional hereditary materials. Meccanica 2013; 48: 1573-1586.

40. Guedes RM. A viscoelastic model for a biomedical ultrahigh molecular weight polyethylene using the timetemperature superposition principle. Polym Test 2011; 30: 294-302.

41. Makris N. Three-dimensional constitutive viscoelastic laws with fractional order time derivatives. J Rheol 1997; 41: 1007-1020.

42. Freed AD and Diethelm K. Fractional calculus in biomechanics: a 3D viscoelastic model using regularized fractional derivative kernels with application to the human calcaneal fat pad. Biomech Model Mechan 2006; 5: 203-215.

43. Lakes RS. The time-dependent Poisson's ratio of viscoelastic materials can increase or decrease. Cell Polym 1992; 11: 466-469. 
44. Mourad A-HI, Fouad H and Elleithy R. Impact of some environmental conditions on the tensile, creep-recovery, relaxation, melting and crystallinity behaviour of UHMWPE-GUR 410-medical grade. Mater Des 2009; 30: 4112-4119.

45. Pegg EC, Murray DW, Pandit HG, et al. Fracture of mobile unicompartmental knee bearings: a parametric finite element study. Proc IMechE, Part H: J Engineering in Medicine 2013; 227: 1213-1223.

46. Zhao D, Banks SA, D'Lima DD, et al. In vivo medial and lateral tibial loads during dynamic and high flexion activities. J Orthop Res 2006; 25: 593-602.

47. El-Domiaty A, El-Fadaly $\mathrm{M}$ and Es Nassef A. Wear characteristics of ultra high molecular weight polyethylene (UHMWPE). J Mat Eng Perf 2002; 11: 577-583.

48. Georgette FS. Effect of hot isostatic pressing on the mechanical and corrosion properties of a case, porouscoated Co-Cr-Mo alloy. In: Quantitative characterization and performance of porous implants for hard tissue applications. Philadelphia, PA: ASTM International, 1987, pp.16.

49. Harrysson OLA, Cansizoglu O, Marcellin-Little DJ, et al. Direct metal fabrication of titanium implants with tailored materials and mechanical properties using electron beam melting technology. Mat Sci Eng 2008; 28: 366373.

50. Liu F, Jin Z, Roberts $P$, et al. Importance of head diameter, clearance, and cup wall thickness in elastohydrodynamic lubrication analysis of metal-on-metal hip resurfacing prostheses. Proc IMechE, Part H: J Engineering in Medicine 2006; 220: 695-704.

51. Landy MM and Walker PS. Wear of ultra-high-molecular-weight polyethylene components of 90 retrieved knee prostheses. J Arthop 1988; 3: S73-S85. 